\title{
Recognizing Complexity: Visualization for Skilled Professionals in Complex Work Situations
}

\author{
Arne W. Andersson, Anders Jansson ${ }^{(\bowtie)}$, Bengt Sandblad, \\ and Simon Tschirner \\ Department of Information Technology, Uppsala University, Uppsala, Sweden \\ anders.jansson@it.uu.se
}

\begin{abstract}
In our research, we study IT-systems for highly skilled professionals in complex and dynamic work situations. Such situations can be found in e.g. health care, process and traffic control and in administration. The demands on the operators/users are often very high concerning quality performance, efficiency, timeliness, safety, communication and cooperation. Our experience shows that human operators can overview, interpret and in real time use an almost unlimited amount of information, if it is relevant to the situation and visualized according to human capabilities. The solution to the visualization problem is therefore not to avoid or hide complexity, but to cope with it, to accept that the complexity must be there. The challenge is to develop systems for visualization and support, which can be used efficiently in relation to the complexity of the work task. We believe in recognizing complexity. First, we describe the scientific foundation of such an approach. Second, we give a detailed example of a complex visualization problem, emphasizing the demanding cognitive operations the operators have to conduct. Finally, we describe the solutions, the visualizations and interactions that make it possible to support the cognitively demanding task, taking care of the complexity without losing the rich amount of information necessary for the operators in different situations, but without adding unnecessary complexity in terms of complicated handling of the user interface and the information systems. Some of these visualizations now run in real systems and have been evaluated, and we end up by suggesting recommendations for successful visualizations in complex work tasks.
\end{abstract}

Keywords: Complex and dynamic work situations - Design of operator systems $\cdot$ Visualization

\section{Introduction}

\subsection{The Research Field}

In our research we focus on the design and use of IT-systems for highly skilled professionals in different types of complex and dynamic work situations. The demands on such experts, e.g. process operators, traffic controllers, health care staff in intensive care units and professionals in more complicated administrative organizations, are 
often very high concerning quality, efficiency, timeliness, safety, communication and cooperation. Our experiences show that organizations and the technologies often are not designed to support skilled professionals in their demanding tasks, and that much of the problems are related to which information that is visualized and how. For different reasons the information systems do not comply with the complexity of the work. In Endsley et al. (2003) this is formulated as: "In the face of this torrent of 'information', many of us feel less informed than ever before. This is because there is a huge gap between the tons of data being produced and disseminated, and our ability to find the bits that are needed and process them together with the other bits to arrive at the actual needed information. That is, it seems to be even harder to find out what we really want or need to know".

To our opinion, the solution to the visualization problem is not to avoid or hide complexity, but to cope with it. We believe in recognizing complexity. The challenge is to design, develop and deploy systems that can be used efficiently despite the complexity of the work tasks. The design of information and control systems, and the user interfaces, must match the complexity, the needs and the capabilities of the human operators.

\subsection{Contents of the Paper}

This paper starts with a specification of the concept complexity in order to be able to explore and discuss it in a systematic way. This is based on recent theoretical progress in the area of cognitive psychology and human-computer interaction. A second concept of importance in our research is dynamics. Here, we also give a definition, following modern literature on dynamic decision making. An example of a design solution is presented, and with its help we describe why human cognition in general is well adapted to complex and dynamic tasks, but only if the design solutions comply with the general principles identified below. We also present a more detailed example of a complex visualization problem, emphasizing the demanding cognitive operations the operators have to conduct. Here, we describe the design solutions, the visualizations and interactions that make it possible to support the cognitively demanding task, without losing the rich amount of information necessary for the operators in different situations, and without adding unnecessary complexity and usability problems. The paper ends with a discussion about why recognizing complexity and understanding the dynamics are important in relation to the operators' understanding of the work domain and control situation.

\subsection{Earlier Studies and Experiences}

We base our results and recommendations on a number of earlier research and development projects. Examples are train traffic control, process control in different organisations, train driving, high speed ship operation, health care systems, home care and administrative work in large organisations. Here we have both analysed existing control systems and operator interfaces and participated in design, development, deployment and evaluation of new systems and interfaces. The work has always been 
very user centred, involving professional operators in all phases of the projects. Our experiences are that traditional models and methods for design of control systems and operator interfaces very often fail in understanding the complexity of the controlled system and the control tasks and do not deliver systems and interfaces that efficiently support skilled operators. We will explain why such pitfalls often are encountered and how they can be overcome. The conclusions and recommendations we present in this paper are on another level than traditional interface design heuristics in humancomputer interaction and guidelines that can be found in literature or in corporate guidelines. Our focus is on understanding and accepting the complexity and to design interfaces which support complex tasks and not add unnecessary usability problems.

\section{Background}

\subsection{Separating Complex Tasks from Complicated Systems}

Let us start with a working definition of complexity. With complexity we here mean the richness and properties of information that is given by the process that is to be controlled, i.e. the essential part of the operator's work. This is not (only) a question of the amount of information, but much more of the properties such as the number of system states, their interdependencies, nonlinearities, time delays, unpredictable behaviour and disturbances, discrete decision possibilities etc. Our view on complexity is in line with Endsley et al. (2003), i.e. separating system complexity from operational complexity and cognitive complexity (display and task complexity). However, we want to separate complexity from dynamics, which is further discussed below. The richness of information is perceived and handled, often with an astonishing level of expertise, by users and operators in different complex and dynamic work situations. Our own experiences in this way relate to train traffic dispatchers, train drivers, high-speed ferry operators, nurses and doctors at intensive care units, process operators in nuclear power industry and paper mills. In recent literature in cognitive psychology, the knowledge that expert users of this kind have is discussed under the heading of intuition. For example, in the recently published and seminal book on human thinking, Kahneman (2011) suggests that there exist two basic conditions for being able to develop expertise and intuitive skills in different kind of work contexts:

1. An environment, or surrounding, that is sufficiently regular in order to be predictable

2. A possibility to learn these regularities through extensive and long-lasting practice

When both these conditions are satisfied, we can expect human operators to develop intuition and expertise based on knowledge and best practice. Such precise judgments have been studied by for example Klein and his associates (Klein 1993; 1998). An experienced car driver can normally, and without high cognitive workload, handle intense rush-hour traffic, manoeuvring the car from one location to another at the other end of the town, and in real time monitoring what is going on inside and outside the car. We monitor and adjust speed and position of the car by controlling gear, brake 
and throttle, observe the behaviour of thousands of other cars, bicycles, pedestrians, traffic signals etc. We can evaluate and take a large number of decisions, sometimes several per second. During this very dynamic and complex process we can, provided that we really are experienced drivers, be cognitively focused on some problem of another kind, e.g. concerning what we are going to cook for dinner or if the newly employed co-worker really is well integrated in our work team. Skilled intuitions, based on recognition-based decisions, seem to develop when the opportunities to learn are generous (Kahneman and Klein 2009). Moreover, Ericsson et al. (2006) have suggested a range of factors that influence the rate of skill development. Among these, the kind of learning people employ, level of engagement and motivation, and the kind of self-regulation processes people use seem to be important. Here, we find a connection to the model for system development we have used in analysing and designing systems in the different domains mentioned above (Tschirner et al. 2014).

Instead of trying to reduce patterns that are rich of information, we should try to include these patterns into the design of modern information systems. This is in line with Norman (2010), who argues that we must learn to live with complexity, not to reduce it. But how do we do that? One problem here is that there seems to be confusion about the use of the term complexity. Many information systems are regarded as complex because their design has failed to adapt to the actual context. But this is to confuse things. A better term for such maladaptive designs is that they are complicated, and that they make things more complicated than necessary. There are numerous examples of complicated systems, caused by e.g. the organization or the design of the information systems. This can make the work more difficult because the operator must focus on the wrong things, e.g. on how to interpret information or how to navigate in the hierarchy of the information system rather than on solving the problems related to the control tasks. Complicated systems of this kind reduce the operator's possibilities to cope with the fundamental complexity of the controlled process.

The design challenge is to accept and not hide the complexity of the work as such, and at the same time not to add unnecessary complexity in the interface.

When we study different work settings, where skilled professionals shall handle very dynamic and complex situations, we see that it is not always easy to understand requirements and find solutions to how the professionals must be supported and how relevant information should be visualized. One purpose of this paper is to discuss this problem in relation to a case study, and we will describe some important aspects related to how information is visualized and used in these situations and the problems and solutions we have identified.

\subsection{The Properties of Dynamic Systems}

Dynamic should not be confused with "in movement", i.e. the contradiction to stationary. Dynamic is the contradiction to static and means that the system has certain important characteristics. A dynamic system e.g. develops over time even if it is not interacted with, it will keep on developing even when the interaction is stopped, and a control action at one time will sometimes not have an immediate effect but is only 
recognizable after some time. In order to understand the current and future behaviour of a dynamic system, it is necessary to know the prehistory of the system and how it has been interacted with. In order to reach a certain desired state in future, it is necessary to know the current state of the system and how to interact with it in a relevant way during some time period. It is necessary to be able to observe the system in enough detail including its history, the goals to be reached must be specified, a model describing the dynamic properties of the system must exist, and there have to be appropriate possibilities to interact with the system. In the case of human control, the model of the system is the operator's mental model. It can easily be understood that much of this requires efficient visualization of information.

In practice, most systems that we are trying to control are dynamic. To manoeuvre a large and heavy ship, to control a nuclear power plant or a paper mill, to plan and control train or air traffic, to monitor the state and give therapy to a patient in an intensive care unit or to handle a complex electronic case in an administrative organisation are examples. In such and many other situations, human operators, marine officers, pilots, process or traffic controllers, physicians, nurses, case handlers etc have the task to monitor, plan and control a complex and dynamic process. For this they require information visualized in an appropriate way. Often this means much, complex and dynamic information. When we study operators' handling of dynamic systems, we base this on a model (Jansson et al. 2014) that explicitly focuses on the models humans develop and the goals they formulate as consequence of the characteristics of the control systems, i.e. the controllability and the observability. Both these properties are important for the ability to develop knowledge on how to approach a system and how to reach desired effects. This model is based on studies on how humans manage dynamic decision tasks (Brehmer 1992). Controllability and observability are always operationalised in some way, i.e., they manifest themselves in some kind of visualization. The visualization is a key factor for the ability of a human operator to handle a dynamic system.

\subsection{An Example}

In this paper we argue that the control of complex and dynamic systems requires complex and dynamic information. Skilled professional operators and users must be supported with information systems that help them to cope with the difficulties they are facing. However, in practice we often see that the developers, in the design of information systems, often try to avoid, reduce, hide or neglect this. The main reason for this is probably both that the designers are not expert enough to understand the complexity of the situation and the needs of the users, and that the users themselves are not aware of the complexity and cannot express their requirements verbally. Another reason can be that the developers deliberately hide the complexity of reasons such as "it will be too complex for the users to handle" or that "it will be too complicated to develop and maintain". The result of not recognizing the complexity will most often result in very poor support for the users and that their possibilities to cope with the challenges in their work tasks are severely limited. 
Our interpretation of this is that, if we reduce or hide the complexity and dynamics that are needed, we cause severe problems for the skilled professionals. They will have problems to work efficiently not because of the complexity of the work, but because the lack of efficient support. In other words, avoiding or hiding complexity will add another type of complexity related to the user interface, and the users will experience an unnecessary cognitive workload. "Too little information will result in cognitive overload". This is a statement that goes in the opposite direction compared to what can be found in the major body of literature on design and visualization today. In Edsley (2003) it is argued that "People can only pay attention to a certain amount of information at once. ... A considerable amount of additional work is required to find what is needed and extra mental processing is required to calculate the information the operator really wants to know. This inevitably leads to higher than necessary workload and error". We have found that people can pay attention to extremely large amounts of information, if relevant and visualized in a way that corresponds with our perceptual and cognitive abilities, e.g. the Gestalt laws.

In the case of train traffic control, see Fig. 1 below, the information on the large screens forms a very complex and dynamic pattern. The skilled traffic controllers can despite this have a very good control of what is going on, without consciously focus on the individual information elements. If something important or unexpected

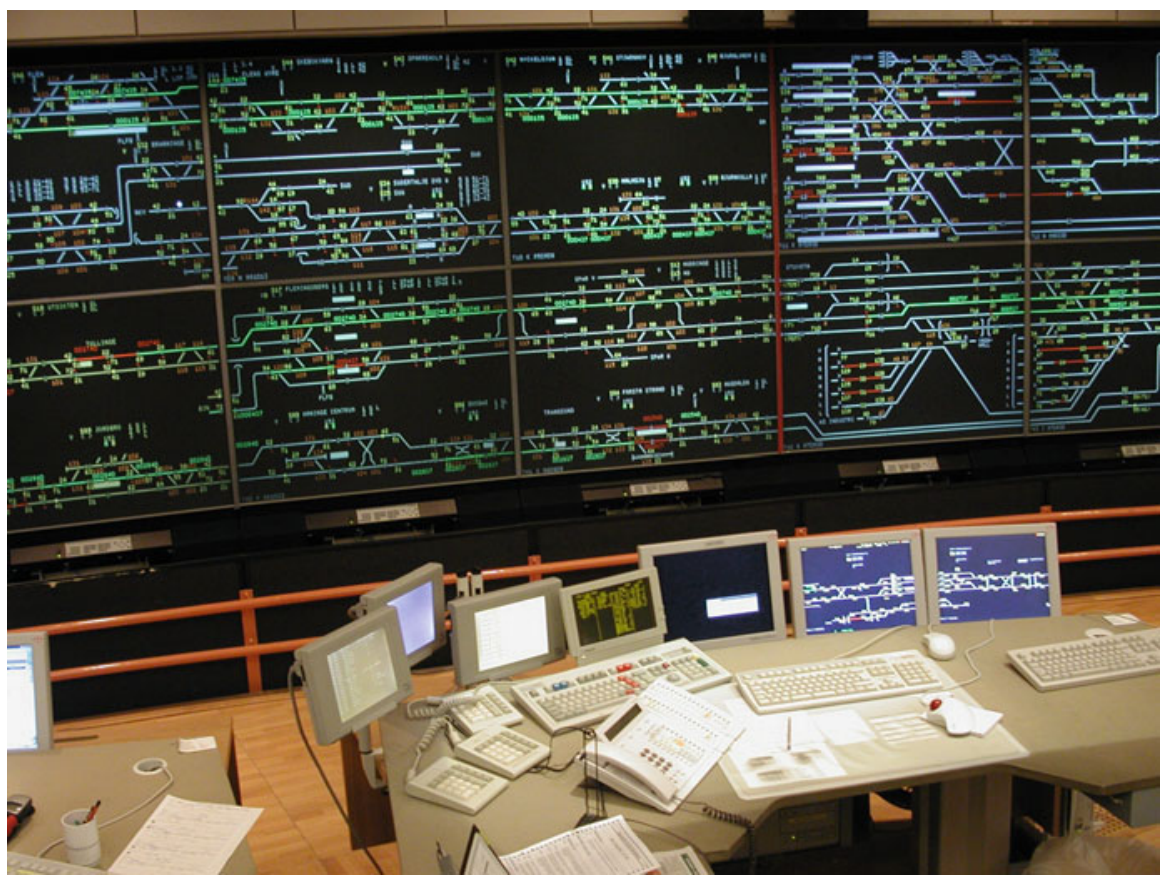

Fig. 1. The information of a very complex process, here in train traffic control in an urban area, forms a pattern that despite its complexity can be perceived and efficiently interpreted by skilled professional traffic controllers. 
happens, they will immediately perceive this and turn their attention to this for further analysis and actions.

Another aspect of this example, which we will discuss more below, is that the complexity shown here is not what they actually need for efficient control. The information visualized here does not reflect the real time dynamics of the train traffic system. To visualize this, other information sets must be a part of the observability for the traffic controllers.

We have found, from several case studies, that skilled professional users in complex work require systems that support them and allow them to use both their advanced professional and their basic human cognitive skills. The users in such work environments also have the normal human cognitive limitations. The design of the system must not come in conflict with these. This makes the design and visualization process very difficult. User-centred design does not mean presenting users with just the information they say they want or need at any given moment (Endsley et al. 2003). To understand the needs of the users and to find an appropriate visualization requires more than traditional user centred models.

\section{Human Cognitive Skills and Limitations}

Today, there is general agreement within research in cognitive psychology that human cognition is well adapted to a natural ecology. Our cognition is efficient and effective in relation to the tasks we are set to handle in such surroundings. This is especially true for what recently has come to be known as System 1 (Kahneman 2011). This system is believed to produce suggestions for interpretations and actions continuously to System 2. This latter system will only be activated if there are reasons to believe that the normal conditions are no longer valid, and that reflective thinking and problem solving is needed. This latter kind of thinking is constrained, mainly by being forced to bring all information to the working memory, which is known to have very limited capacity. Thus, when we argue that too little information will give cognitive overload, it is because the working memory has to continuously check and scan a new dialogue or a new window with information, without being able to relate the different presentations to each other in a semantically meaningful way. The design solutions are in these cases not adapted to the requirements of System 1, which immediately activates System 2 and prompts for a solution. System 2 reacts to the situation in its own way, which is considerably slower, by allocating a lot of attention to this new task, with the result that other on-going cognitive processes are interfered with and interrupted.

System 1 is associated with low level processing, high parallel capacity and low cognitive constrain, but also with rapid and efficient pattern recognition, and use of Gestalt laws. It has almost an unlimited capacity to overview, perceive and interpret information, also in real-time. On the down side, System 1 comes with a number of consequences in terms of biases (Tversky and Kahneman 1974; Kahneman and Tversky 1996), but as mentioned above, it is believed that operators of the kind we study are able to develop expertise and intuitive skills without exhibiting these biases, since they deal with a sufficiently regular and predictable environment and are using 
the possibility to learn about these regularities through extensive practice (Kahneman and Klein 2009). However, this is true if, and only if, the visualization of the information is well adapted to the needs of the operators. If not, the support system will be perceived as making things more complicated, often inducing the need to call System 2 into work. System 2 is limited in capacity and very sensitive to different kind of disturbances. When using working memory, it is often System 2 that has to carry out these processes.

\section{A Case Study: Train Traffic Control}

The train traffic system of Sweden is geographically divided into eight control areas. Train traffic in each area is controlled from a centralized traffic control centre (TCC). At the TCC, information about the traffic process status is presented in track diagrams on large distant panels, see Fig. 1 above, and/or on several computer screens at the workplace, see Fig. 2 below. Operators, the traffic controller here called dispatchers, monitor the train movements and control train routes by automatic or manual remote blocking systems. Track usage is controlled either by automatic functions or by manually specifying and executing interlocking routes for each track section. Today's control systems are often designed to support the operator's possibilities to react on and to solve disturbances and conflicts when they occur. In order to meet increasing future demands, new principles and technical solutions are required for efficient train traffic control. Controllers should be able to follow the dynamic development of the

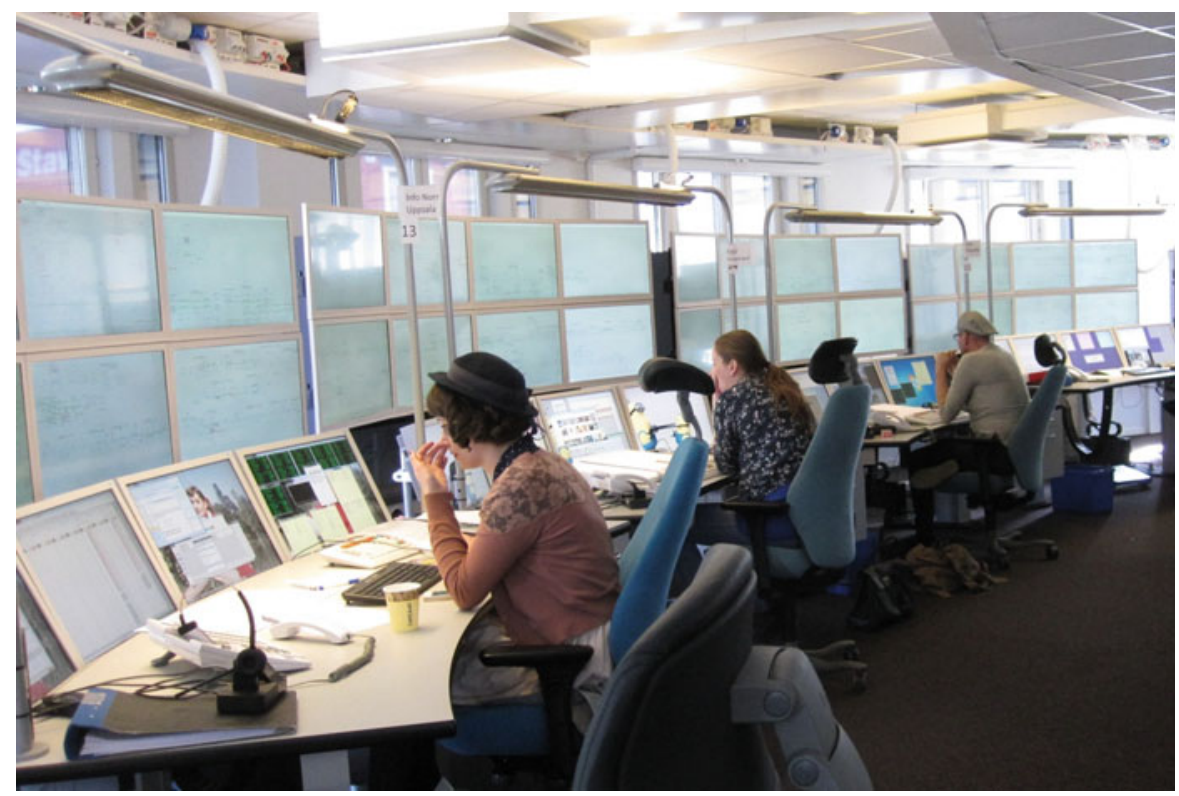

Fig. 2. The same control centre as in Fig. 3, but here re-designed for visualization using a number of large size screens at each individual workplace. 
traffic system over time and rather prevent disturbances than solving them when they already have caused delays.

This project has been a long research cooperation between our department and the Swedish Transport Administration. It started with a deep analysis of the present systems for traffic control and the problems experienced there. Requirements and prototypes of new systems and interfaces were successively developed and evaluated in close cooperation with groups of experienced traffic controllers and managers. After detailed laboratory experiments a full scale test systems was developed, deployed and tested in two different traffic control centres. The evaluations have shown that the new systems, based on the type of recommendations presented in this paper, contributed to radically improved performance. The professional traffic controllers were supported to move from control of the technical infrastructure to control of the traffic flow and to more optimal handling of traffic perturbations and disruptions (Sandblad et al. 2010).

One main objective has been to shift the control paradigm from low-level technical control tasks into higher level traffic re-planning tasks (Kauppi et al. 2006). Re-planning tasks must be supported by efficient user interfaces that allow the train traffic controller to be continuously updated and able to identify and evaluate present and future traffic conflicts so that these can be taken care of in time. The traffic controller must have high situation awareness (Endsley 1996). Improving train traffic control is a very cost effective way to better utilization of existing infrastructure. The traffic controller can be pro-active and prevent conflicts.

When the traditional traffic control work was analysed, several interesting and important findings related to visualization were made. Some of these are the following.

Today's control systems are designed to support the operator's possibilities to react on disturbances and to solve problems and conflicts when they have occurred. A paper-based time-distance graph is used as a tool for planning. Train dispatchers change the traffic plan by drawing new time table lines in the graph. The paper graph is then used by the dispatcher to remember what needs to be done and at which time. Since the new traffic plan is not automatically introduced into the system, there is great risk that the different automatic functions in the system will work against the new plan. Automatic support systems are not predictable enough to the dispatchers, because of their internal complexity. Automates can cause automation surprises by performing control actions that contradict the dispatchers plan. Bainbridge (1983) called this "the irony of automation", that when workload is high, automation is of the least assistance. To avoid automation surprises, train dispatchers are often forced to take full control by inhibiting all automatic functions in the disturbed area and solve the situation manually. Planning is mainly a mental process and the dispatcher must remember the plan without support and give control commands at the appropriate time. This causes a high cognitive workload and disturbs problem solving.

When decision making of the traffic controllers was analysed, we found that the information they base their decisions on was not directly available on the panels and screens. They had to observe what was available and then cognitively generate the information they actually need. This is an extremely complex cognitive task and requires advanced mental models and long experience. One concrete problem is that 
the exact position and speed of the trains are not visualized, since this information is not available from the signalling system. The only information available is the occupation of a track segment, indicated as red on the panel. By observing the shift from one segment to the next, the controllers "know" the position, speed and identification of each train. If the train suddenly stops, this is not visualized but will after some time be understood by the controllers since the red segment does not shift as expected.

The controllers' understanding of what dynamically happens, past, present and future, is not based on any visualization of dynamic information. Instead the controllers must remember what happened earlier, sometimes by writing things down, measuring time delays using a stop watch and perform control actions without any support that shows predictions of future events.

To summarize, the control of this extremely complex and dynamic system is today not supported by relevant and usable information systems and the visualization, although rather complex, does not show any dynamic or directly decision relevant information.

A project was initiated where researchers and a group of experienced dispatchers worked together, in a very user centred way, to describe and analyse today's systems and problems, to develop a new control strategy and to design prototypes of new, better information systems. A special focus was on aspects related to complexity and understanding of the dynamic properties of the train traffic control system.

The result was a completely new control principle and a completely new visualization of information to the dispatchers (Kauppi et al. 2006). The new control principle means that the dispatchers, i.e. the traffic controllers, will be supported by completely new systems for re-planning, traffic control and visualization. In the new system they are supported to monitor the dynamic movements of all trains in their control area, to identify disturbances and traffic conflicts and efficient tools to solve these conflicts by re-planning directly in the interface. The traffic plan is executed automatically when the planned train movements are approaching present time. An example of the new interface is shown below in Fig. 3. The re-planning tools (Fig. 4) and some details on indication of traffic conflicts (Fig. 5) are also shown below.

The new control system and traffic controller user interface have been fully developed and evaluated in full scale operational traffic control at two control centres in Sweden. Evaluations have shown that the concept and design contribute to improved support to the dispatchers and better planning of train traffic (Sandblad et al. 2007).

The most important differences and improvements achieved, compared to the old control principles and operator interfaces, are:

- The dispatchers, i.e. the traffic controllers, can now focus on identifying the disturbances and conflicts and make appropriate changes in the traffic plan. This is supported by visualizing decision relevant information and supporting re-planning. The execution of control commands is completely automated, something that earlier was a main task and took cognitive capacity from the problem solving activities. 


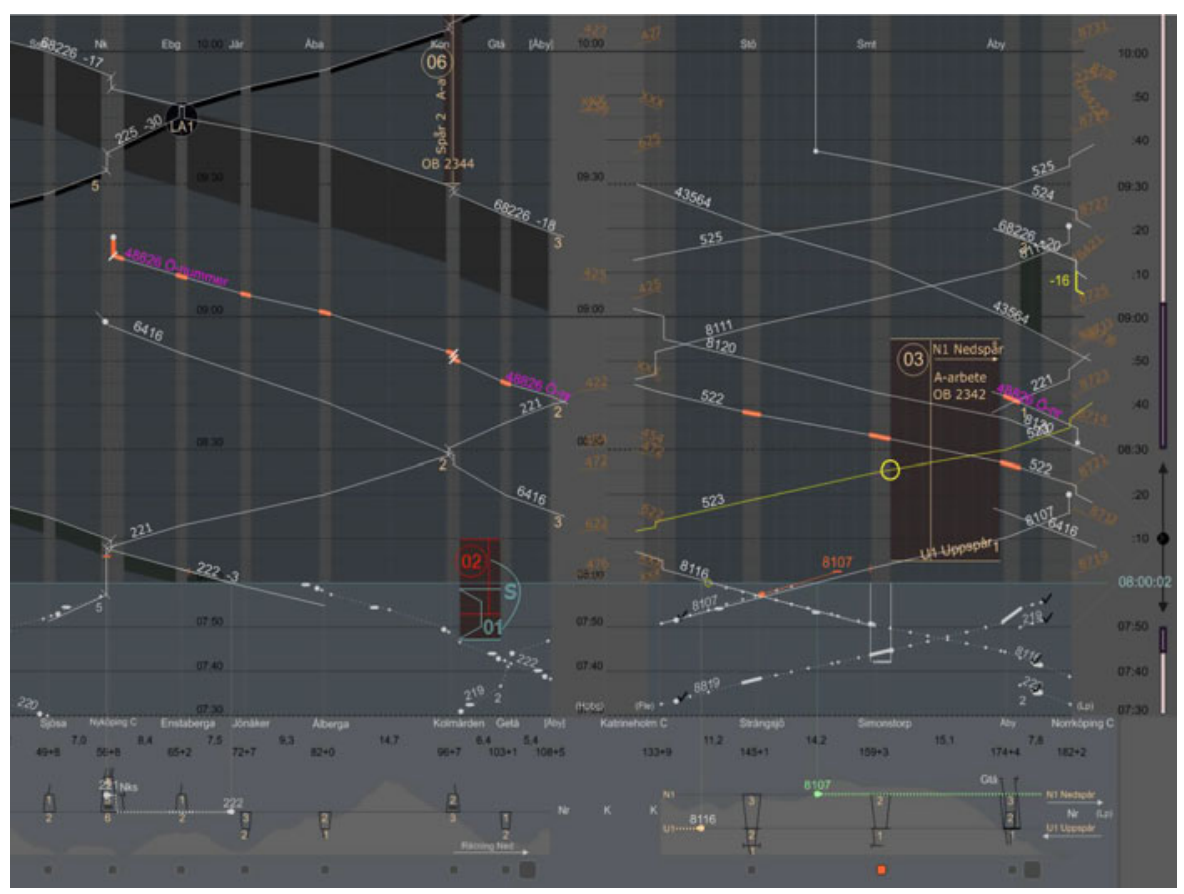

Fig. 3. The new traffic controller user interface. The interface shows the train traffic plan, deviations from the original plan, the planning via a time-distance graph, the present state (train position and speed), the history, planned maintenance work etc. Static information is e.g. the track structure.

- The interface shows history, present time and future plans. In this way the dynamic properties of train movements and signalling system are continuously visualized.

- The interface shows all decision relevant information simultaneously and in realtime, which minimizes unnecessary cognitive workload.

- The effects of re-planning actions are always directly visible, so the dispatchers have a direct feed-back on their actions.

- The traffic plan can be made available to all involved actors in the traffic process, e.g. train drivers and railway companies, which minimizes the need for oral communication.

\section{Recommendations}

Experiences from several applied research projects that we have been involved in or have detailed knowledge about, together with the more theoretical background described above, allow us to make some more general conclusions and recommendations. 


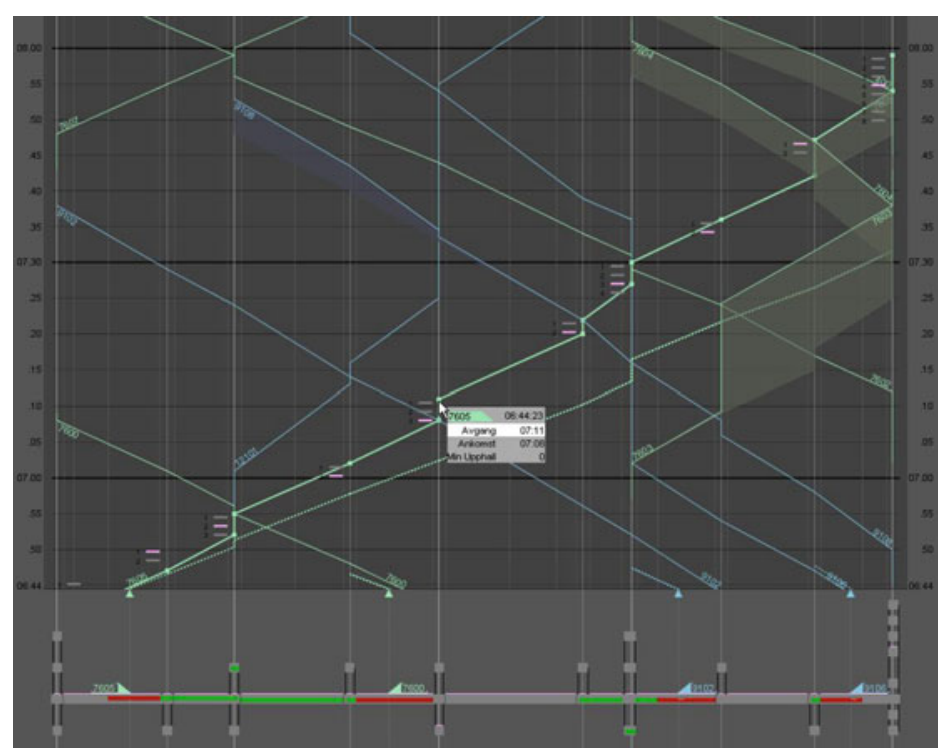

Fig. 4. The re-planning tasks are performed directly in the graph. A train is selected and highlighted. The selected graph line can be manipulated using the mouse scroll wheel. In this way all re-planning tasks can be performed directly in the interface and the resulting plan is directly visible. Examples of re-planning tasks are changing departure and arrival times, track usage, train speed etc.

\subsection{Recognizing Complexity in Design of Visualization}

If a work situation is complex, and if the process that is controlled is complex and dynamic, then the human operators will depend on complex information to perform their tasks. It is never a good idea to hide the complexity or to try to be "user friendly" by simplifying things in a way that does not support skilled professional users. Skilled professionals rely on complex information to perform their work. Therefore, the design of an operator system must be based on how we can support the operator's work in an efficient way, recognizing complexity and solving the conflict between complexity and good design.

"Cognitive overload" most often comes from visualizing too little or irrelevant information and of using bad coding and design. If well designed, the human operator can overview, interpret and handle very large, almost unlimited, amounts of information. If the information needed for the complex and dynamic tasks is not visualized in an appropriate way, the human operator must develop and use very advanced mental models and the very limited capacity of the short term memory. This will cause "cognitive overload". Already very small amounts of information can be impossible to use if not properly designed. Information overload in a complex and dynamic situation comes from showing too little, not too much! 


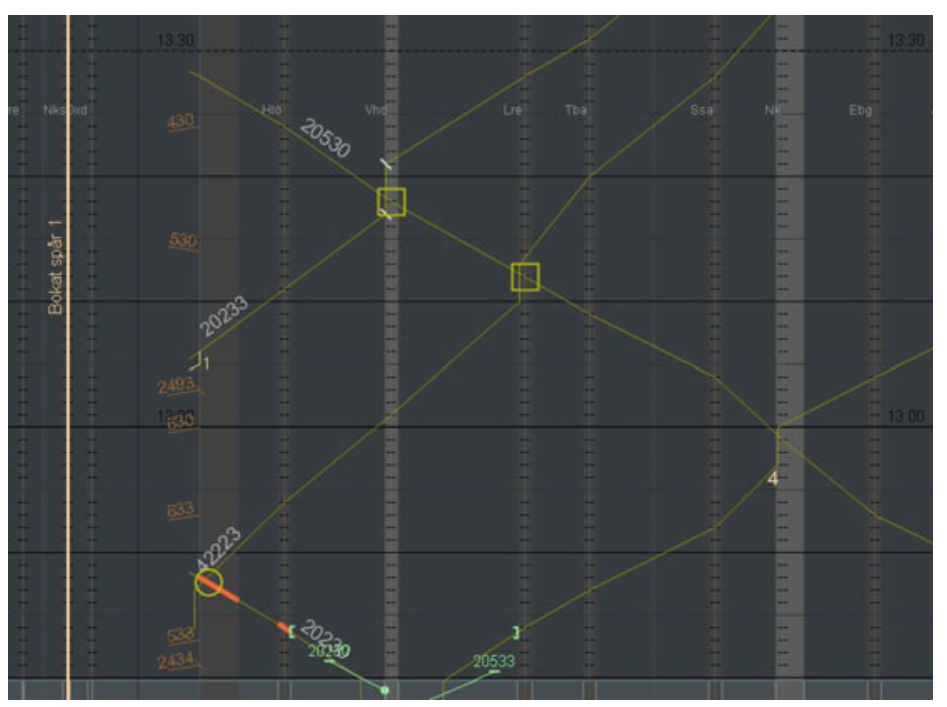

Fig. 5. Example of visualization of different types of conflicts. Conflicts are automatically detected and indicated. Yellow circles here mean a line conflict, e.g. two trains meet on a single track line. A yellow square means station track conflict, i.e. two trains are planned to use the same station track. During re-planning the removal or appearance of a conflict is visualized in real time (Color figure online).

\subsection{Recognizing Complexity in the Systems Development Process}

The process of analysing the control process and work situation, specifying requirements, designing, developing and deploying the control system and the visualization and interaction in the operator interface must be based on very detailed knowledge about the work performed by the operators. The process must be user centred and actively involve skilled professional users. Traditional process, task analysis and modelling methods can neither capture the complexity of the information the operators need, nor details or requirements concerning visualization and interaction. It is only using iterative user centred methods, prototyping and evaluations that can fulfil the actual demands.

\subsection{Recommendations for Visualization}

We can, based on the experiences discussed above, give some concrete practical recommendations concerning visualization in complex and dynamic control situations.

Different types of recommendations, guidelines and heuristics for design and visualization of operator interfaces have been presented earlier. In Endsley et al. (2003) a set of recommendations for design of systems and interfaces to support high situation awareness are described. Our recommendations below are to a large extent in compliance with these, but are more concrete and aimed to support the actual visualization. 
We must here emphasize that it is never possible to give general recommendations that can be directly applied in the design of a specific system in a specific context. The detailed solution will always "depend on...". But based on the general recommendations, together with necessary knowledge about how to interpret and apply these, the following examples can support the design of usable interfaces for humans in control of complex and dynamic systems.

Below we present some of the most important recommendations.

Show the Whole and the Details Simultaneously. Rationale: If the user/operator has to focus on a specific detail, it is important to show the relation of this detail to the rest of the available information. In many systems the information can either be shown in form of an overview or (and only or) in form of a selected detail. When a specific detail is visualized, the user cannot see the relation of the detail to the whole and cannot see which other details are available and how to find them. In such systems it is easy to get lost and to fail to notice or consider important things that occur in other parts of the system. At least it will add cognitive load that disturb the chain of thoughts.

Example: In an electronic patient record (EPR) system all information about one patient is stored. Very often the EPR contains very large amounts of information related to different medical problems, encounters, episodes and time periods. In an old paper based patient record the physician could hold the whole record in one hand and turn pages with the other. The whole was always visible when a certain page was opened. Today the physicians often report that they lack overview, easily get lost in the record, that they always are afraid to miss important information and that they are slow in reading and spend most of their time searching and navigating. If the whole is always visible, these problems can to a large extent be avoided (see Figs. 6, 7).

Show all Information Needed Simultaneously; Support Continuous Overview of the Whole Process. Rationale: Based on the fact that we humans have an ability to overview, perceive and interpret almost unlimited amounts of information simultaneously and in real-time, if it is properly visualized, we have found that it is often possible to show very large amounts of information simultaneously. This is especially true for skilled professional operators in a complex work environment. When the pattern formed by the information is well known and relevant to the goals of the work, large sets of information can be monitored and analysed with very low cognitive load. This is not surprising since we humans are used to be and act in complex and dynamic environments when we e.g. drive a car in rush hour traffic or walk around in the town on a busy Saturday, meet friends in the crowd and watch for things to buy in the shop windows. If we do not see all information simultaneously, we must start to remember things in our limited working short term memory and use cognitive capacity for navigation etc.

The human operator needs to be continuously aware of what is going on, in order to be prepared to act when needed and to be able to act proactively. Even if the information visualized is very complex, an experienced operator can, if the design supports this, easily scan the images whenever needed and directly without conscious attention identify relevant perturbations or disruptions. If not all relevant details are continuously available, the operator has to actively search for relevant information, 


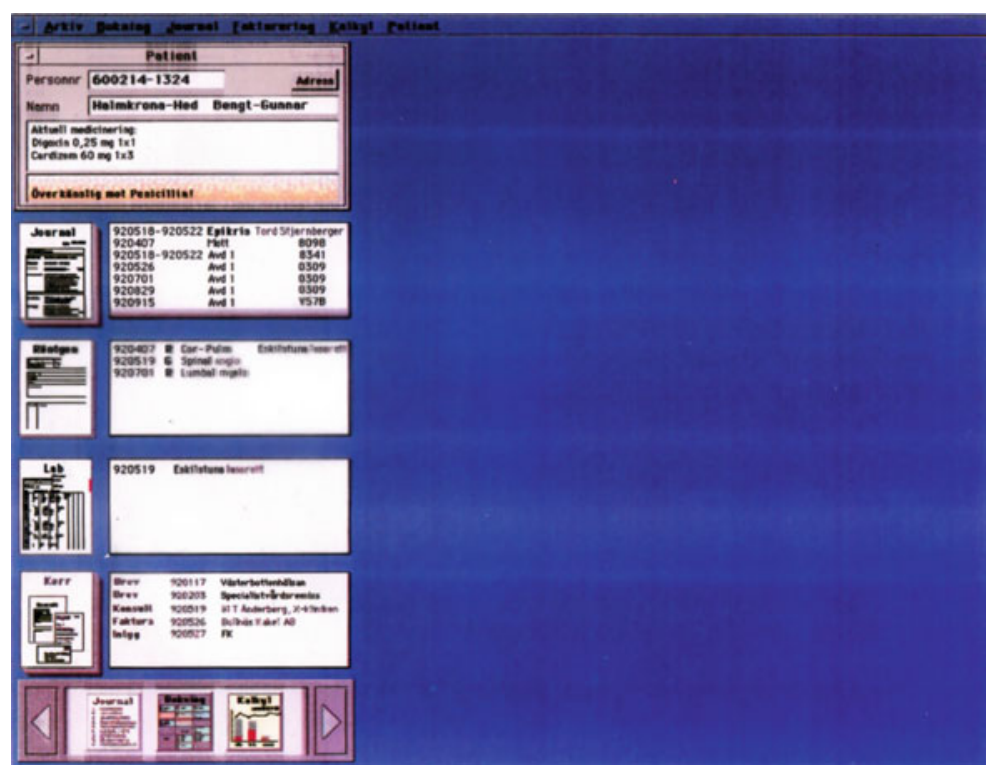

Fig. 6. In this example an overview of the total EPR is visualized. It consists of a patient card with important data that are always visible and four piles of documents together with an index of each pile. The user can choose to open one pile or select a specific document from the index. The menu at the bottom is for navigation to other information "rooms".

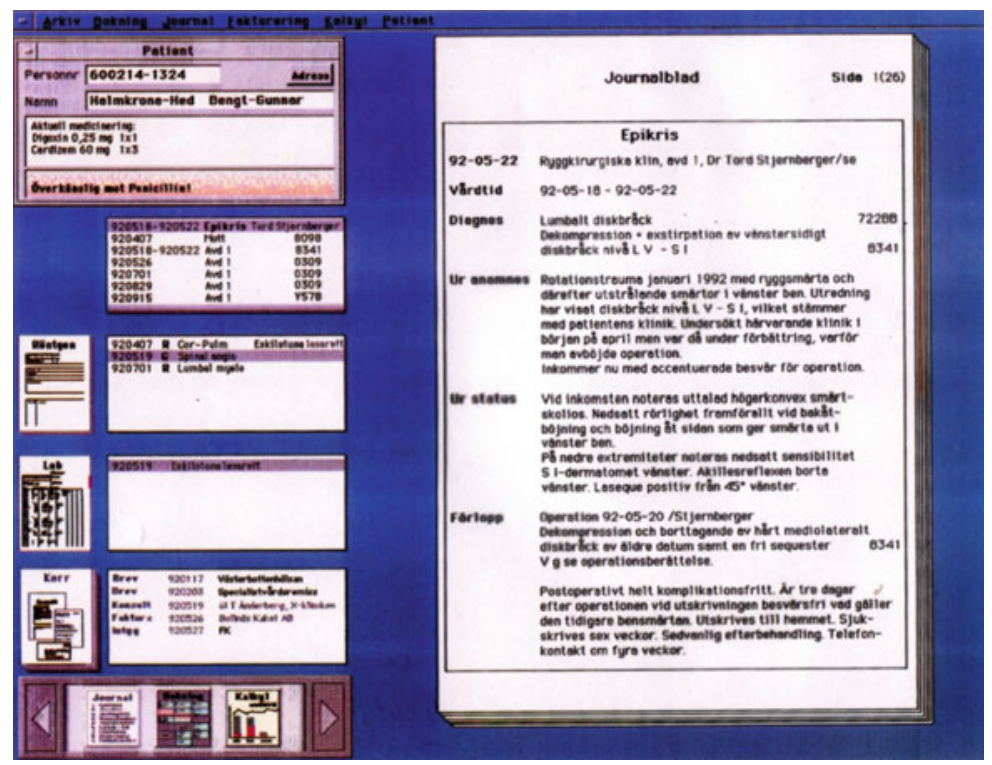

Fig. 7. Here one document is opened for reading. The whole record is still visible and it is easy to identify which pile and document is opened. Reading of consecutive pages is done by turning of the pages. Links between different documents are indicated in the indexes. 
something that requires high level attention, makes it easy to miss important things, can lead to safety problems etc.

This also includes the information needed for development of efficient mental models. If the operators continuously can follow the dynamic behaviour of the whole system they will learn to understand the dynamic properties of the system and how they can and should act in different situations.

Example: Figure 8 shows an example of an operator user interface from a process industry. Here the whole process is visible in one single view, together with diagrams showing the dynamic development of different process states. The development of the process can be observed continuously and important changes can be identified and taken care of before they develop into something problematic. The amount of information is not a problem for the operator.

This could be compared to another design, where the operator was supposed to monitor an overview where only some states were observable, together with eventual alarms. When needed, the operator could select a process detail showing one part of the process. In this case there were more than 20 different details available. When one detail was selected, the whole and the other parts are not visible.

In Fig. 3 above, we can see another example. Here the whole train traffic and signalling process, including history and planned future, can be displayed in one single view. When this new traffic controller interface was presented, even experienced controllers were fist afraid that this was too much information to overview and interpret in real-time. Our argument, as being the designers, was mainly that this is actually the information they use today in their decision making and control tasks, but without seeing it. If they do not see the information they must develop extremely complex mental models to handle the situation. When implemented, the controllers rather fast learned to use the interface efficiently and the evaluation indicated that they developed much better understanding of the complexity and dynamics of the traffic process and could improve the control.

Show Dynamic Information. Rationale: Operators of dynamic systems are always interested in the development of the system. They want to know what is going on, what probably will happen, how they can control the system so that a desired behaviour or state is reached etc. If they only see the present state of the system, but are interested in the history, the trend or how fast the state is changing, they have to actively remember or even take notes in order to follow the dynamics. For an experienced process operator the key information are the first and second time derivatives, not (only) the present value. If dynamic data are visualized, the operator can by just scanning over the image get a direct feeling for what is going on.

Example 1: See Fig. 9.

Example 2: In Fig. 8 above, the relevant dynamic information for different process states are visualized. The dynamics of the system states can be easily observed by the experienced operator and unwanted behaviour detected before anything gets critical. This supports high situation awareness and pro-active control. It has shown to be a common main task for process operators to act so that the risks for problematic situations are reduced. To have continuous high situation awareness, and to take care 


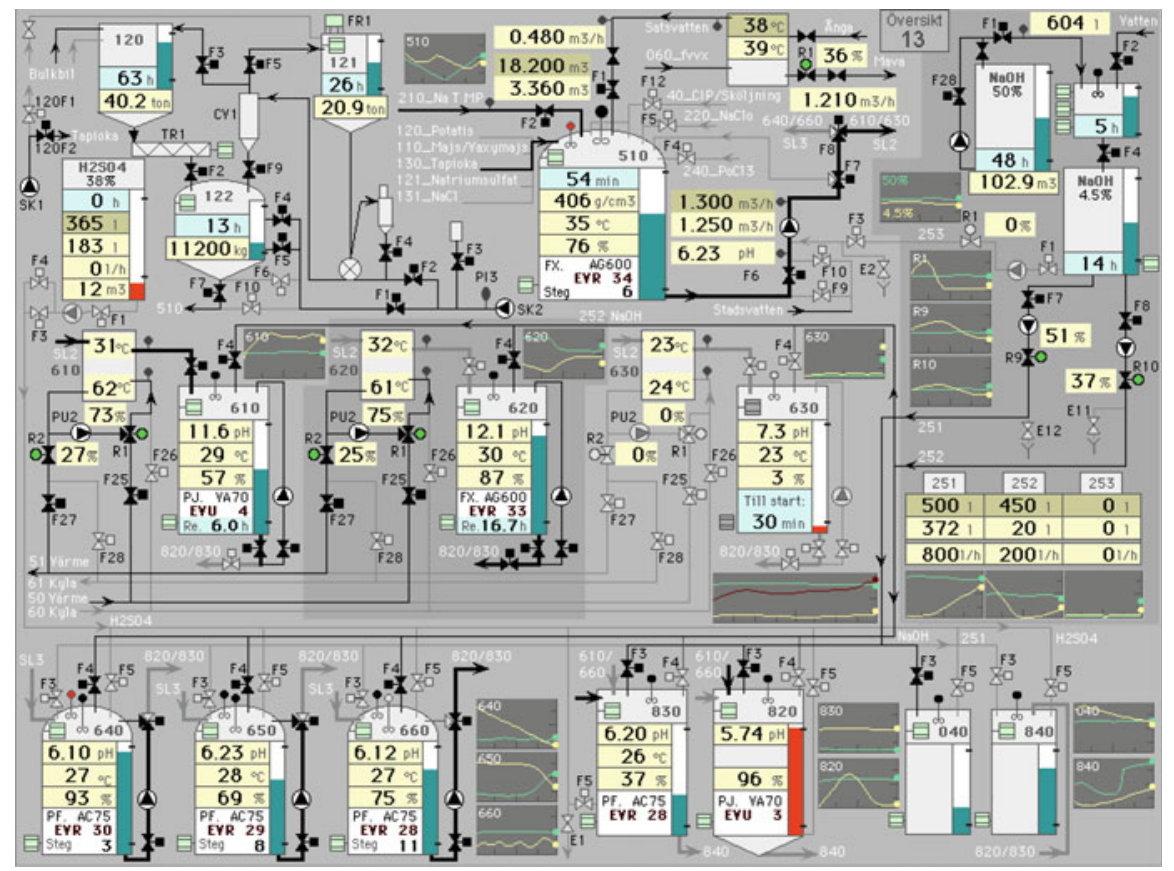

Fig. 8. Example of an operator interface for industrial process control. The visualization contains both static information in the background and dynamic information in the foreground. Information is coded with regard to the importance for the operator. Despite its complexity, for novice users, a skilled operator has no problems to overview and interprets the interface using very low cognitive capacity.

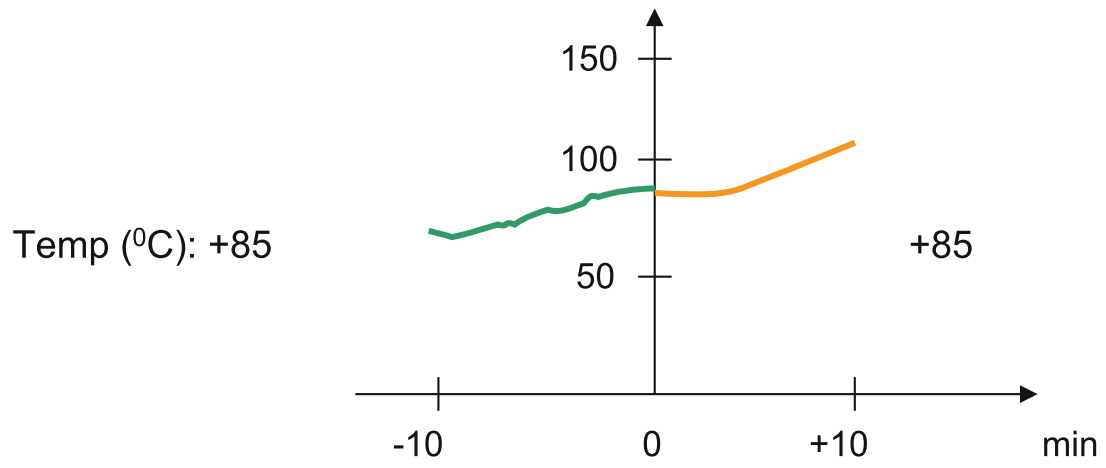

Fig. 9. The temperature can be rather uninformative, while the history and trend are much more relevant for the operator. If the temperature just now is $85{ }^{\circ} \mathrm{C}$, this can have a very different meaning and require different actions, depending on if it is sinking or rising, and how fast. 
of perturbations as early as possible, reduce the risk to have to handle chaotic situations later.

Emphasize what is Important. Rationale: In complex work environment, when very large sets of dynamic information are visualized, it is important to support identification of what is important in a certain situation. What is important in a given context must be thoroughly investigated and visualized efficiently. Static structures can be of less importance. Data values (e.g. 85) are more important than entities (e.g. ${ }^{\circ} \mathrm{C}$ ). High values can in one context be more important than low ones etc.

Example: See Fig. 8 above. Here very many items are coded in relation to their importance. The static structure that the operator always knows is in the background. Important process parts, states, values etc. are in the foreground. Importance is coded by e.g. contrast, font size, colour etc. Using a medium grey background allows coding using both light (white as background coding) and dark (black as foreground coding) colours.

Time Related Information. Rationale: It is often important for the user to relate information to a time scale. The information as such can only be interpreted in relation to when it was generated and in relation to other data. The information must be visualized in a form that the user/operator easily can relate to a time scale.

Example 1: In train traffic control, the traditional way to present information was by track diagrams (see Fig. 1 above). If a certain track section was occupied by a train, the section was coloured red. But it was not indicated when the section became occupied and when is can be expected to be free again. In Fig. 3 above an alternative visualization is used. All train movements are visualized in form of lines in a timedistance graph. This allows the traffic controller to see the dynamics of the system and relate track occupation to time. The information can be easily interpreted even for large train traffic regions.

Example 2: In the patient record system it can be important for the user, physician or nurse, to be able to relate e.g. laboratory findings, patient encounters, events of medical relevance etc to a time scale. When such presentations are not shown, the users often complain that they "do not see the process and cannot relate different data to each other". This can e.g. be shown by the option to select a view where relevant data are visualized along a lime axis.

Show Effects of Alternative Decisions. Rationale: In the control of complex and dynamic systems the operator often has to evaluate complex situations and decide on what to do and when. Often it is also difficult to predict the possible outcomes, on short and long term, of alternative decisions. One solution can be to allow the operator to try different alternative solution to the present problem and to directly visualize the result of these solutions. This requires that it is possible to separate the decision from the execution, so that the operators are given the possibilities to "play around" before making the final decision.

Example: One example can be seen in Fig. 4. Here the train traffic controller is allowed to try different alternatives for re-planning of the traffic before selecting one. When a certain decision is tried, all consequences such as upcoming conflicts, delays of trains etc. are directly visualized. 
Support Development of Mental Models. Rationale: Normally the operator's user interface is designed to support control tasks. We know that an important part of the work of the controller is to continuously further developing their mental model of the controlled system. Even if certain dynamic properties are not directly needed for control, they are essential for development of the mental model.

Example: Show dynamic information continuously. Allow the operator to "play around" and see the effects of manipulations. See Fig. 4 above.

Efficient Coding of Information. Rationale: Visualizing large sets of dynamic data requires efficient coding. Based on theory of perception (properties of the human sight/view/eye system) and cognition, many heuristic rules for efficient coding can be derived. What is a good solution must be evaluated in each specific context.

Example: Different coding mechanisms can be used. Examples are: contrast, use of colours, foreground-background, patterns instead of text, fixed positions, consistency etc.

Visualization of Automatic Systems. Rationale: Automation very often adds complexity to the operator's work. If automatic systems are autonomous, i.e. do not only execute the operators' intention but make decisions according to their own algorithms, it becomes difficult for the human operator to predict what the automatic systems will do, when and why. This will cause "automation surprises" (Bainbridge 1983) and other problems. One common result is the "turn-it-off" syndrome, i.e. the operators turn the automatic system off in order to be in full control.

Example: One solution can be to only use non-autonomous automatic systems, so that they only execute the operator's plans. If autonomous systems are used, it becomes important to visualize what the automatic systems will do, when and why. This can in many cases be extremely difficult.

\section{Discussion}

We have argued that skilled human operators, in complex and dynamic work situations, must be efficiently supported. This requires that they are provided with systems, interfaces and visualization that help them to cope with the challenges. The solution is not to hide, avoid or neglect the complexity, but to accept it and find appropriate ways to support the skilled professional users. We have found, with support from theories and from a number of case studies, that skilled human operators can handle extremely complex and dynamic situations without cognitive overload. On the contrary, limiting the complexity and showing too little information can often cause cognitive overload since this will cause lack of the information the operators need for understanding and deciding on control actions. Here the visualization is a real challenge. We must efficiently visualize very large, complex and dynamic information patterns. The information presented to the operators/users must be designed to support human and professional skills, without interfering with our inherited limitations or adding unnecessary workload.

Classical heuristics of HCI are too general to guide design of control systems and operator interfaces in very complex and dynamic work situations. The recommendations presented in this paper try to complement traditional design knowledge in this respect. 
When we base the design of operator interfaces on the recommendations above, we have found that the operators' work is better supported and that acceptance from the operators is high. They report that they now "have an overview and know what is going on". They can be continuously in-the-loop, have high situation awareness and develop a pro-active behaviour.

\section{References}

Bainbridge, L.: Ironies of automation. Automatica 19(6), 775-779 (1983)

Brehmer, B.: Dynamic decision making: human control of complex systems. Acta Psychol. 81, 211-241 (1992)

Endsley, M.R.: Automation and situation awareness. In: Parasuraman, R., Mouloua, M. (eds.) Automation and human performance: theory and applications, pp. 163-181. Lawrence Erlbaum, Mahwah (1996)

Endsley, M.R., Bolte, B., Jones, D.G.: Designing for Situation Awareness: An Approach to Human-Centered Design. Taylor \& Francis, London (2003)

Ericsson, K.A., Charness, N., Hoffman, R.R., Feltovich, P.J. (eds.): The Cambridge Handbook of Expertise and Expert Performance. Cambridge University Press, New York (2006)

Jansson, A., Tschirner, S., Andersson, A.W., Sandblad, B.: Trading rigor for relevance: GMOC - a conceptual model for cognitive field studies. Unpublished manuscript (2014)

Kahneman, D.: Thinking. Fast and Slow. Allen Lane/Farrar, Straus and Giroux, London/New York (2011)

Kahneman, D., Klein, G.: Conditions for intuitive expertise: a failure to disagree. Am. Psychol. 64(6), 515-526 (2009)

Kahneman, D., Tversky, A.: On the reality of cognitive illusions. Psychol. Rev. 103, 582-591 (1996)

Kauppi, A., Wikström, J., Sandblad, B., Andersson, A.W.: Future train traffic control: control by re-planning. Cognition Technol. Work 8(1), 50-56 (2006). Springer-Verlag London Ltd.

Klein, G.A.: A recognition-primed decision (RPD) model of rapid decision making. In: Klein, G.A., Orasanu, J., Calderwood, R., Zsambok, C.E. (eds.) Decision Making in Action: Models and Methods, pp. 138-147. Ablex, Norwood (1993)

Klein, G.: Sources of Power: How People Make Decisions. MIT Press, Cambridge (1998)

Norman, D.: Living with Complexity. MIT Press, Cambridge (2010)

Sandblad, B., Andersson, A.W., Kauppi, A., Wikström, J.: Implementation of a test system for evaluation of new concepts in rail traffic planning and control. In: Wilson, J., Norris, B., Clarke, T., Mills, A. (eds.) People and Rail Systems. Human Factors at the Heart of the Railways. Ashgate Publ. Comp, Abingdon (2007)

Sandblad, B., Andersson, A.W., Kauppi, A., Isaksson-Lutteman, G.: Development and implementation of new principles and systems for train traffic control in Sweden. In: Ning, B., Brebbia, C.A., Tomii, N. (eds.) Computers in Railways XII, pp. 441-450. WIT-press, Southampton (2010)

Tschirner, S., Jansson, A., Andersson, A.W., Sandblad, B.: GMOC - a conceptual model for analysis and design of human-work interaction. Unpublished manuscript (2014)

Tversky, A., Kahneman, D.: Judgments under uncertainty: Heuristics and biases. Science 185, 1124-1131 (1974) 